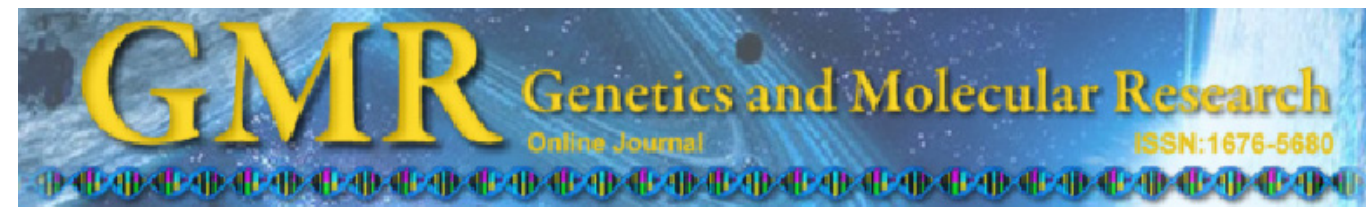

\title{
Interactions between rare-earth ions and DNA of Bashibai sheep
}

\author{
J. Aniwashi ${ }^{1,2 *}$, H.A. Kaleri ${ }^{1,3 *}$, Y. Sulaiman ${ }^{2}$, L. Qi-fa ${ }^{1}$ and X. Zhuang ${ }^{1}$ \\ ${ }^{1}$ College of Animal Science and Technology of Nanjing Agricultural University, \\ Nanjing, China \\ ${ }^{2}$ College of Animal Science, Xinjiang Agricultural University, Urumqi, China \\ ${ }^{3}$ Department of Animal Breeding \& Genetics, \\ Faculty of Animal Husbandry and Veterinary Sciences, \\ Sindh Agriculture University, Tando Jam, Pakistan \\ *These authors contributed equally to this study. \\ Corresponding author: X. Zhuang \\ E-mail: zxie@njau.edu.cn
}

Genet. Mol. Res. 10 (2): 1075-1083 (2011)

Received September 20, 2010

Accepted December 30, 2010

Published June 7, 2011

DOI 10.4238/vol10-2gmr1070

\begin{abstract}
The interaction between rare-earth ions and DNA from Bashibai sheep was studied by microcalorimetry and electrochemistry. The DNA chain was found to have four to five binding sites for rareearth ions. The binding affinity was about $10^{-5}-10^{-6} \mathrm{M}$. It was also found that smaller ions caused more heat to be released in the process of binding and bound more readily to the nucleic acid chain. This is attributed to the enhanced ability of polarization of smaller ions and reduced steric hindrance compared to larger ions. The electrochemistry results show that rare-earth ions could be inserted into the DNA helix, producing a new complex with electrochemically active groups. The rare-earth ions and DNA complex reached equilibrium after a 90-min incubation at room temperature.
\end{abstract}

Key words: Rare earth; Nucleic acid chain; Microcalorimetry; Bashibai sheep 


\section{INTRODUCTION}

China has a centuries-old history of breeding sheep. It has abundant sheep breed resources, with more than 40 local sheep breeds, which serve as important genetic resources for the sustainable development of animal husbandry and for the preservation of biological diversities. For example, Ganjia and Oula sheep in Gansu Province belonging to local breeds of Tibetan sheep can adapt to the plateau environment and endure coarse feeding ( $\mathrm{Du}, 2003$; Wang et al., 2006). The Bashibai sheep, which is raised in Xinjiang Province, has delicate and tasty meat that is easier to digest and has a higher calcium and iron content (Wang et al., 2007). In addition, there are other local sheep breeds, such as Henan big-tail sheep and Heiqiupi sheep, which possess unique features. Previous studies on these breeds were carried out only at the morphological level. There are still no reports on some of the breeds at the molecular level.

The purpose of this study was to investigate the genetic diversity and phylogenetic evolution of Chinese sheep based on the analysis of the complete sequence of the Cyt $b$ gene. This will be helpful for the conservation, utilization, and exploitation of the genetic resources of indigenous Chinese sheep.

Because trace rare-earth elements can promote the growth of plants (Chen et al., 2001), they are added to fertilizer, which is widely used in agricultural production. As a result, rare-earth elements occur in almost every corner of the Chinese mainland. The rare-earth elements were characterized with regard to their physiological functions and biological effects on some organisms (Peng and Wang, 1991; Zhu et al., 1998; Zhao et al., 2000). After they get into the living body, however, what effect they have on organism and how they take part in metabolism remain unknown. In recent years, growing concern has been expressed about rare-earth elements, because they are a potential threat to the environment. In the long run, rare-earth elements may be a new kind of pollution.

DNA is a long linear polymer that carries information in a form that can be passed from one generation to the next. Genetic information is stored in the sequence of bases along a nucleic acid chain. Thus, the stability of DNA is very important for the safety of species (Jeremy et al., 2001). Rare-earth ions have positive charges and a 4f-electron orbit, which have an affinity for negative charges and $\mathrm{N}, \mathrm{O}, \mathrm{P}$ atoms on the nucleic acid chain. With the accumulation of rare-earth ions, their impact on the stability of DNA should be evaluated, on which there is no previous report. In this study, both microcalorimetry and electrochemistry were used to study the affinity of rare-earth ions for DNA from Bashibai sheep and how rareearth ions bind to the nucleic acid chain.

\section{MATERIAL AND METHODS}

Analytical reagent grade lanthanum nitrate ( $\mathrm{La}, \mathrm{Ce}, \mathrm{Eu}, \mathrm{Ho}, \mathrm{Yb}$ ) was obtained from the China Medicine (Group) Shanghai Chemical Reagent Corporation.

\section{Samples tested}

We selected 189 healthy sheep randomly at a Bashibai sheep center for producing sheep, Yumin County, Xinjiang, consisting of 40 white line, 40 black line, 34 orange line, and 75 lean new line. Ear tissue samples were taken in $1.5-\mathrm{mL}$ centrifuge tubes containing $70 \%$ ethanol and were kept at $-20^{\circ} \mathrm{C}$ in the laboratory for future use. 


\section{Main reagents}

TaqDNA polymerase and dNTP mixture were purchased from TaKaRa Biotechnology Ltd.; proteinase K, Tris, EDTA, SDS, N, N'-methylene bisacrylamide, TEMED, ammonium persulfate, agarose, and acrylamide from Lanzhou Pengcheng Biotechnology Co., Ltd., and Tris-saturated phenol, chloroform, isoamyl alcohol, silver nitrate, and anhydrous ethanol were of analytical grade.

DNA was extracted from samples and detected by the method described previously (Sambrook, 1989).

\section{DNA quality and concentration determination}

Quality testing of genomic DNA was checked by $0.8 \%$ agarose gel electrophoresis, UV lamp observation, and preliminary analysis of DNA extraction efficiency. Genomic DNA concentration was determined with a UV spectrophotometer; optical density was measured at absorbances of 260 and $280 \mathrm{~nm}$, and the DNA concentration $(\mathrm{ng} / \mu \mathrm{L})$ was calculated as follows: DNA concentration $=50 \times \mathrm{OD}_{260} \times$ dilution factor $/ 1000$. Pure DNA $=\mathrm{OD}_{260} / \mathrm{OD}_{280}$ $\approx 1.8$ (a ratio greater than 1.9 indicates that there is RNA contamination; a ratio less than 1.6 indicates the presence of protein, phenol and other pollutants).

\section{Microcalorimetry}

TAM Air (Thermometric AB, Sweden), which is an 8-channel heat conduction microcalorimeter for measurements of heat flow, was designed to monitor continuous heat released or absorbed in a series of processes. The performance and the details of this instrument have been previously described (Xie et al., 1988; Peng and Wang, 1991; Peng et al., 2003). The instrument was improved to perform the titration experiment. With the buffer in the reference ampoule unchanged, the sample ampoule was installed with the titration setting, as shown in Figure 1 . The titration setting contains a syringe, one reservoir, one vent, and some tubules to connect them. When performing an experiment, the reference ampoule is filled with buffer, which has the same heat capacity as the sample. The syringe and reservoir are then filled with titrant, and the titration ampoule is filled with the material to be titrated. The calorimeter is then equilibrated to the desired temperature.

Prior to the experiment, the electric calibration and chemical calibration were performed on the instrument at $25^{\circ} \mathrm{C}$. According to the manual, the electric calibration was performed by a permanent precision heated on each channel. The reaction between the diluted hydrochloric acid and a large excess of diluted sodium hydroxide was used as chemical calibration in this instrument. After subtraction of dilution heat of each injection, the enthalpy of reaction can be calculated.

At 298.15 K, with the reference ampoule filled with buffer, the sample ampoule was filled with $10 \mathrm{~mL} 10^{-5} \mathrm{M}$ DNA dissolved in Tris- $\mathrm{HCl}$ buffer. The syringe and reservoir were filled with titrant $-5 \times 10^{-4} \mathrm{M}$ rare-earth ions. After the titration settings were put into the calorimeter, $2 \mathrm{~h}$ were spent to equilibrate to the same temperature of the thermostat. The titration experiment was performed by injection of rare-earth ions into the DNA solution in the sample ampoule. 


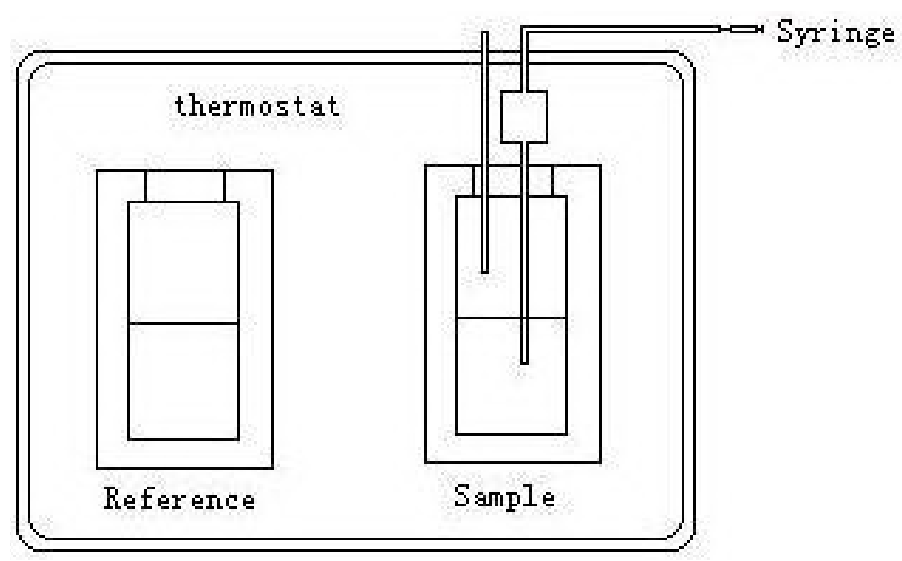

Figure 1. Schematic diagram of the isothermal titration device on TAM Air.

\section{Electrochemistry}

All the electrochemical experiments were performed in a conventional three-electrode cell, a glassy carbon working electrode, an $\mathrm{Ag} / \mathrm{AgCl}$ (saturated $\mathrm{KCl}$ ) reference electrode, and a platinum wire counter electrode. Cyclic voltammetries were performed in a CHI660B electrochemical work station (CHI Instruments, Shanghai, China).

Prior to the experiments, the work electrode was polished by $1.0,0.3,0.05 \mu \mathrm{m} \alpha-\mathrm{Al}_{2} \mathrm{O}_{3}$, then washed with distilled water, and acetone for $5 \mathrm{~min}$. High-purity nitrogen was used to deaerate the solutions for at least $10 \mathrm{~min}$. After $\mathrm{Ce}^{3+}$ was incubated with DNA for different times, the voltammograms were recorded with cyclic potential scan at $20 \mathrm{mV} / \mathrm{s}$. The voltammograms were recorded with the titration of $1.125 \times 10^{-6} \mathrm{M}$ DNA into a stock solution of $10^{-4} \mathrm{M} \mathrm{Ce}^{3+}$. The voltammograms were recorded in the mixture of $1.125 \times 10^{-6} \mathrm{M} \mathrm{DNA}$ and $10^{-4} \mathrm{M} \mathrm{Ce}^{3+}$ with different scanning speeds. All experiments were typically carried out at room temperature.

\section{RESULTS AND DISCUSSION}

At $298.15 \mathrm{~K}$, rare-earth ions were titrated into DNA solution in Tris-HCl buffer. The thermogenic curve in the titration process is shown in Figure 2. The proposed binders show a concentration dependence on the measured heat from the microcalorimetric titration experiments, while a non-binder essentially shows only effects associated with heat of dilution and all peaks will be of the same size during repeated injections. The integrated heat of each injection is shown in Figure 3, from which it could be seen that heat was produced accompanying the binding of incremental additions of rare-earth ions to DNA.

According to the principle of isothermal titration calorimetry, the titration setting was applied for determination of binding interaction. With the help of a non-linear curve fitting carried out by the Microcal Origin software, the binding thermodynamic parameters, $\mathrm{K}, \mathrm{n}$, and $\Delta_{r} H_{m}^{\ominus}$ can be calculated from actual calorimetric data (Freire et al., 1990; Qu et al., 2002). The Gibbs free energy $\Delta_{r} G_{m}^{\ominus}$ and entropy $\Delta_{r} S_{m}^{\ominus}$ of the binding reaction can be obtained by Equations 1 and 2 . 


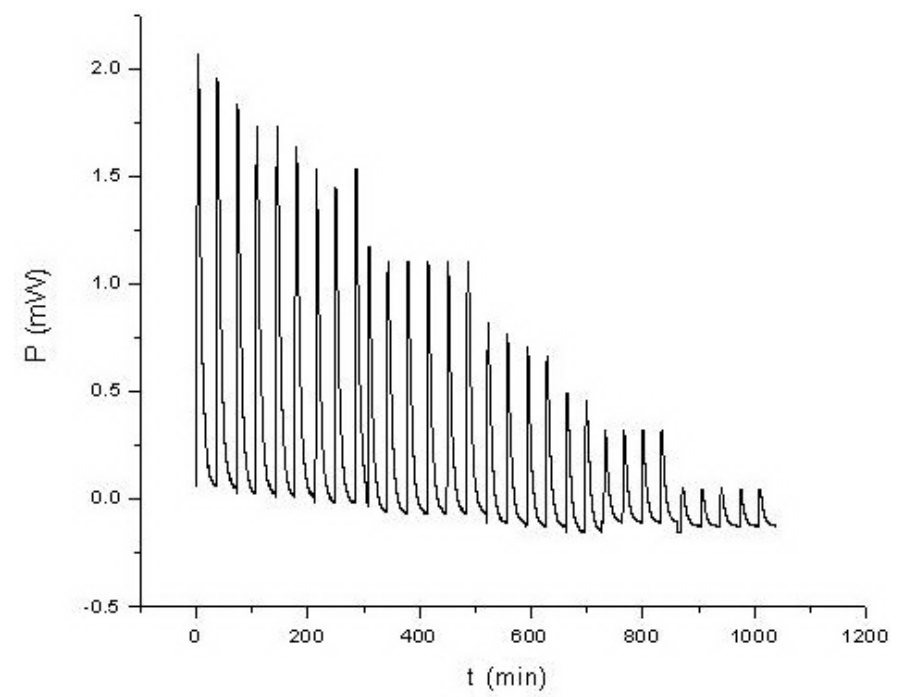

Figure 2. Thermal curves for rare-earth ion being titrated in native DNA solution.

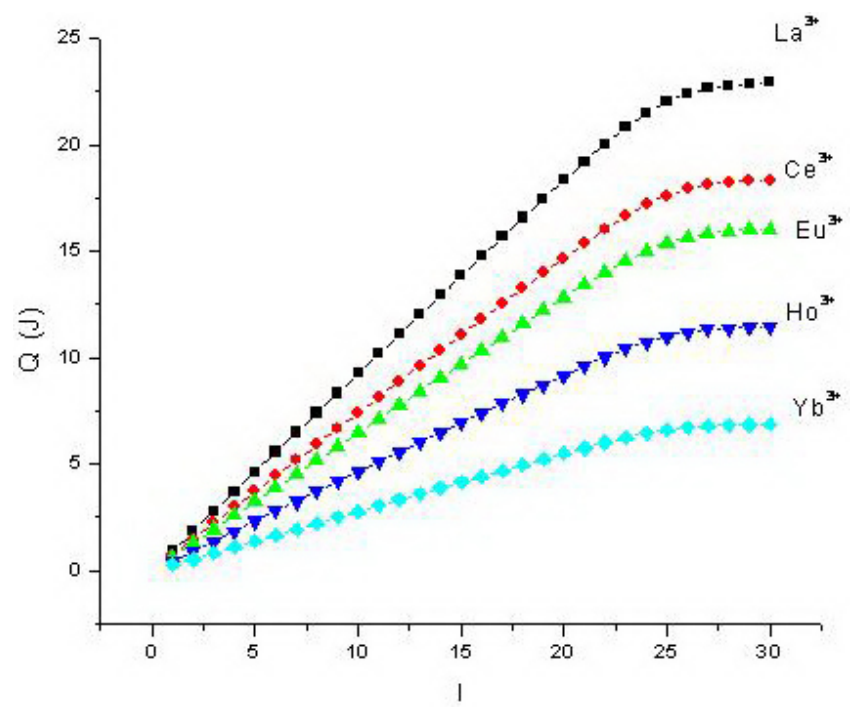

Figure 3. Calorimetric curve of rare-earth ion binding to native DNA.

$$
\Delta_{r} G_{m}^{\Theta}=-R T \ln K
$$

$$
\Delta_{r} G_{m}^{\Theta}=\Delta_{r} H_{m}^{\Theta}-T \Delta_{r} S_{m}^{\Theta}
$$

The analysis of the calorimetric curve was performed with the help of non-linear curve fitting of Microcal Origin. The binding affinity (K), binding stoichiometry (n) and binding thermodynamics $\left(\Delta_{r} H_{m}^{\ominus}\right)$ could be calculated and are shown in Table 1. 


Table 1. Thermodynamic parameters for binding reaction of rare-earth ions and DNA.
\begin{tabular}{lccccc} 
& $\mathrm{n}$ & $\mathrm{K} \theta$ & $\Delta_{r} H_{m}^{\ominus}(\mathrm{kJ} / \mathrm{mol})$ & $\Delta_{r} G_{m}^{\ominus}(\mathrm{kJ} / \mathrm{mol})$ & $\Delta_{r} S_{m}^{\ominus}(\mathrm{J} / \mathrm{mol})$ \\
\hline $\mathrm{La}^{3+}$ & 3 & $6.38 \times 10^{5}$ & -57.05 & -33.13 & -80.22 \\
$\mathrm{Ce}^{3+}$ & 3 & $8.66 \times 10^{5}$ & -58.46 & -33.88 & -82.40 \\
$\mathrm{Eu}^{3+}$ & 4 & $1.07 \times 10^{6}$ & -60.89 & -34.41 & -88.80 \\
$\mathrm{Ho}^{3+}$ & 4 & $3.92 \times 10^{6}$ & -61.42 & -37.63 & -79.78 \\
$\mathrm{Yb}^{3+}$ & 5 & $6.34 \times 10^{6}$ & -63.55 & -38.82 & -82.93 \\
\hline $\mathrm{n}=$ binding stoichiometry; $\mathrm{K}=$ binding affinity; $\Delta_{r} H_{m}^{\ominus}, \Delta_{r} G_{m}^{\ominus}, \Delta_{r} S_{m}^{\ominus}$ (see page 4 for details).
\end{tabular}

According to the analytical results of titration experiments, the DNA has 4-5 binding sites for rare-earth ions. The binding affinity is about $10^{-5}-10^{-6} \mathrm{M}$. From $\mathrm{La}^{3+}$ to $\mathrm{Yb}^{3+}$, the ion radii decrease gradually. More changes in enthalpy and Gibbs free energy for heavy rare-earth ions were observed compared to light rare-earth ions. The results suggest that the smaller ions cause more heat released in the process of binding and are easier to bind to the nucleic acid chain. The association force between DNA and small ions has covalent bond, hydrogen bond, electrostatic attraction, Van der Waals attraction, hydrophobic force, and so on. In this case, covalent bond and electrostatic attractions are the main force, in the binding process. As to the electrostatic attraction, the high valence and small ion size favor the formation of ionic bonds (Yang, 1991). Besides, a small ion size also favors the formation of covalent bond because of the steric hindrance of large ions on the long polymers. Therefore, the smaller rare-earth ions have higher affinity for the nucleic acid chain.

All the reactions in this experiment showed negative values of $\Delta_{r} H_{m}^{\ominus}$ and $\Delta_{r} S_{m}^{\ominus}$ in the binding process. The standard enthalpy $\Delta_{r} H_{m}^{\ominus}$ can be considered an indicator of the increase in intermolecular bond energies in binding process, while the standard entropy $\Delta_{r} S_{m}^{\ominus}$ reflects the decrease in disorder of the system during the reaction process. In the process of electrostatic self-assembly, the cations migrated from the three-dimensional solution to the DNA surface and were fixed by the association force. The degree of disorder was inevitably reduced and the entropy of the system decreased, which went against the adsorption process. However, the exothermal change of electrostatic self-assembly was observed, which is helpful for the occurrence of the reaction. With the changes of enthalpy and entropy in this experiment having opposite contributions, the binding of rare-earth ions and DNA is an “enthalpy-driven" reaction (Paola et al., 1994; Peng et al., 2007; Liu et al., 2008).

At the molecular level, microcalorimetry has been widely applied in the understanding of the mechanism of regulation and control of biological processes. Its advantage lies in the direct determination of heat signal occurring in the binding process and avoiding the need to partition between the free and bound ligands. Because nearly all binding interactions are accompanied by a change in enthalpy, and because all reactions of interest will produce a thermal signal, calorimetry offers the possibility of directly determining not only the binding constant, and thereby free energy, but also the stoichiometry, enthalpy, and entropy in a single experiment.

The electrochemical characteristics of metal complexes are found to affect possibly their DNA-binding properties (Moriwaki et al., 2008; Millonig et al., 2009). Many intercalators of DNA such as ethidium, daunomycin, etc., are good electron acceptors, whereas all bases and base pairs of isolated DNA, e.g., adenine, thymine, and adenine-thymine, etc., are good electron donors.

Figure 4 shows the cyclic voltammetries of $\mathrm{Ce}^{3+}$ with the titration of DNA into a stock solution. There is an oxidation-reduction of $\mathrm{Ce}^{3+}$ in the graph. With the addition of DNA, peak 
current increased correspondingly and there was no new oxidation-reduction reaction. Also, the oxidation peak shifted a little. This phenomenon suggested that $\mathrm{Ce}^{3+}$ bound to the DNA chains and both of them produced the new complex with electrochemical active groups $(\mathrm{Xu}$ and Bard, 1995). The new complex could respond on the work electrode and the peak current increased. The positive oxidation peak suggests that $\mathrm{Ce}^{3+}$ was inserted into the nucleic acid chain. Figure 5 shows the cyclic voltammetries of the mixture of $\mathrm{Ce}^{3+}$ and DNA with different incubation times. The same phenomenon was observed as in Figure 4. With longer incubation time, the peak current increased and the oxidation peak shifted positively. It suggested that the new complex with electrochemical properties could be produced without an electric field in the process of binding of $\mathrm{Ce}^{3+}$ to DNA chains. This binding is also involved in a dynamic process. After $90 \mathrm{~min}$, the substances in solution would reach an equilibrium.

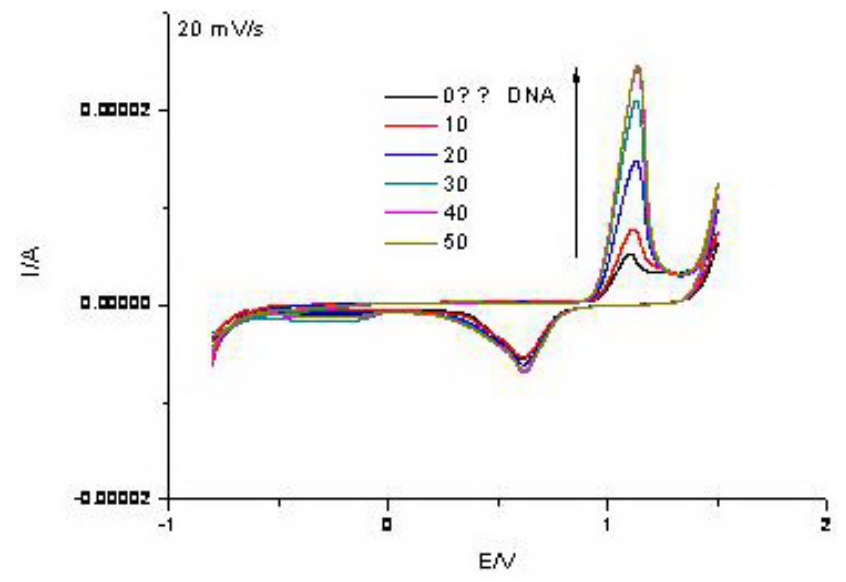

Figure 4. Cyclic voltammetry curves of $\mathrm{Ce}^{3+}$ with the titration of DNA in stock solution with a scanning speed of $20 \mathrm{mV} / \mathrm{s} . \mathrm{C}_{\mathrm{Ce} 3+}=10^{-4} \mathrm{M} ; \mathrm{C}_{\mathrm{DNA}}=1.125 \times 10^{-6} \mathrm{M}$.

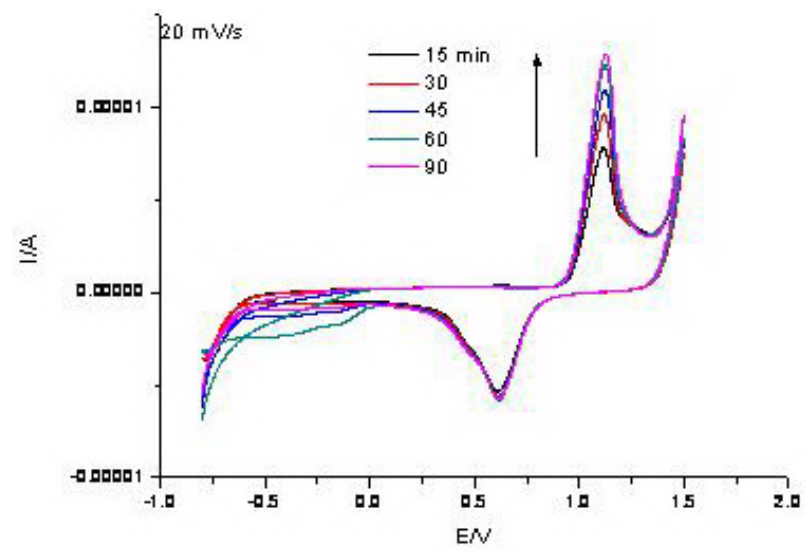

Figure 5. Cyclic voltammetry curves of mixture of $\mathrm{Ce}^{3+}$ and DNA with different incubation times and scanning speed of $20 \mathrm{mV} / \mathrm{s} . \mathrm{C}_{\mathrm{Ce} 3+}=10^{-4} \mathrm{M} ; \mathrm{C}_{\mathrm{DNA}}=1.125 \times 10^{-6} \mathrm{M}$. 


\section{CONCLUSION}

The interaction between rare-earth ions and DNA from Bashibai sheep was studied by microcalorimetry and electrochemistry. Due to covalent bonds and electrostatic attraction, rare-earth ions have a high affinity for DNA chains with a binding constant $10^{-6} \mathrm{M}$. The smaller ions could bind to the DNA chains more tightly. This is attributed to enhanced ability of polarization of smaller ions and reduced steric hindrance compared with larger ions. Thus, the heavy rare-earth ions are more likely to bind to DNA. The electrochemistry results show that rare-earth ions could be inserted into the DNA helix and a new complex with electrochemical active groups was produced. In mixture, rare-earth ions, DNA and the new complex could reach an equilibrium after a 90-min incubation at room temperature.

\section{ACKNOWLEDGMENTS}

Research supported by the National Natural Science Foundation of China (\#30671503) and Programs for Scientific and Technological Department Development of Xinjiang Uygur Autonomous region (\#200431101) and Key Project for Scientific and Technological Department Development of Xinjiang Uygur Autonomous region (\#200731135).

\section{REFERENCES}

Chen C, Zhang P and Chai Z (2001). Distribution of some rare earth elements and their binding species with proteins in human liver studied by instrumental neutron activation analysis combined with biochemical techniques. Anal. Chim. Acta 439: 19-27.

Du L (2003). Several reasons on the appreciation and preservation of Chinese local livestock and birds. China Anim. Husbandry Vet. Med. 30: 3-5.

Freire E, Mayorag OL and Straume M (1990). Isothermal titration calorimeter. Anal. Chem. 62: 950A-959A.

Jeremy MB, John LT and Lubert S (2002). DNA, RNA and Flow of Genetics Information, Biochemistry. 5th edn., W.H. Freeman and Company, New York, 117-119.

Liu P, Li X and Pan M (2008). Thermodynamic studies of electrostatic self-assembly of poly diallyldimethylammonium chloride on proton exchange membrane. Chin. J. Chem. 26: 1215-1218.

Millonig H, Pous J, Gouyette C, Subirana JA, et al. (2009). The interaction of manganese ions with DNA. J. Inorg. Biochem. 103: 876-880.

Moriwaki H, Osborne MR and Phillips DH (2008). Effects of mixing metal ions on oxidative DNA damage mediated by a fenton-type reduction. Toxicol. In Vitro 22: 36-44.

Paola G, Valeria F, Gilli G and Borea PA (1994). Enthalpy-entropy compensation in drug-receptor binding. J. Phys. Chem. 98: $1515-1518$

Peng A and Wang WH (1991). Environmental Bioinorganic Chemistry. Peking University Press, Beijing, 137.

Peng L, Yi L, Jia H, Fengjiao D, et al. (2003). Microcalorimetric investigation of the effect of $\mathrm{La}^{3+}$ on mitochondria isolated from avian chicken liver tissue cells. J. Therm. Anal. Cal. 73: 843-849.

Peng L, Hongyu X, Xi L, Lifang R, et al. (2007). Calorimetric study of nonspecific interaction between lead ions and bovine serum albumin. Biol. Trace Elem. Res. 118: 97-103.

Qu SS, Liu Y, Wang TZ and Gao WY (2002). Thermodynamics of binding of cadmium to bovine serum albumin. Chemosphere 46: 1211-1214.

Sambrook J, Fritsch EF and Maniatis T (1989). Molecular Cloning: A Laboratory Manual. 2nd edn. Cold Spring Harbor Laboratory Press, New York.

Wang X, Ma YH and Chen H (2006). Analysis of the genetic diversity and the phylogenetic evolution of Chinese sheep based on Cyt b gene sequences. Yi Chuan Xu. Bao 33: 1081-1086.

Wang X, Ma YH, Chen H and Guan WJ (2007). Genetic and phylogenetic studies of Chinese native sheep breeds (Ovis aries) based on mtDNA D-loop sequences. Small Ruminant Res. 72: 232-236.

Xie CL, Tang HK, Song ZH, Qu SS, et al. (1988). Microcalorimetric study of bacterial growth. Thermochim. Acta 123: 33-41.

Genetics and Molecular Research 10 (2): 1075-1083 (2011)

CFUNPEC-RP www.funpecrp.com.br 
$\mathrm{Xu} \mathrm{XH}$ and Bard AJ (1995). Immobilization and hybridization of DNA on an aluminum (III) alkanebisphosphonate thin film with electrogenerated chemiluminescent detection. J. Am. Chem. Soc. 117: 2627-2631.

Yang P (1991). Introduction of Bioinorganic Chemistry. Xi'an Jiaotong University Press, Shaanxi, 182-281.

Zhao RM, Liu Y, Xie Z, Shen P, et al. (2000). A microcalorimetric method for studying the biological effects of $\mathrm{La}^{3+}$ on Escherichia coli. J. Biochem. Biophys. Methods 46: 1-9.

Zhu B, Zhao DQ, Ni JZ, Ying D, et al. (1998). Lanthanide binuclear macrocyclic complexes as synthetic enzymes for the cleavage of DNA. J. Mol. Catal. A Chem. 135: 107-110. 